\title{
ARTICLES \\ COMBINING QUOTA-SHARE AND EXCESS \\ OF LOSS TREATIES ON THE REINSURANCE OF $n$ INDEPENDENT RISKS
}

\author{
By Lourdes Centeno and Onofre Simões \\ ISEG, Universidade Técnica de Lisboa, Portugal
}

\begin{abstract}
In this paper, we seek to find the optimal retentions for an insurance company which intends to reinsure each of $n$ risks belonging to its portfolio, by means of a pure quota-share treaty, a pure excess of loss treaty or any combination of the two. The criterion chosen to the selection of the optimal programme is the maximization of the adjustment coefficient, attending to the relationship existing between this coefficient and Lundberg's upper bound of the ruin probability.
\end{abstract}

\section{INTRODUCTION}

Suppose that an insurance company seeks reinsurance for $n$ independent risks (by a risk we mean a single policy or a group of policies-so we could speak of $n$ independent lines of insurance), and has a choice between a pure quota-share treaty, an excess of loss treaty or any combination of the two, for any of the risks. The way this combination operates is as follows: first the quota share contract will apply, so that the insurer shall remain responsible for no more than its share - established by the contract - of any claim that may occur for that risk; afterwards, the excess of loss contract applys, so that, by no means, shall the insurer (of course considering only that part for which it remains reponsible after the quota-share contract) pay more than a certain fixed amount of any claim that takes place.

The problem consists of determining the optimal retention limits for each risk, in each of the two forms of reinsurance. "Optimal" in the sense those limits maximize the adjustment coefficient and, therefore, minimize the upper bound to the ruin probability, supplied by Lundberg's inequality. This same criterion was also adopted by WATERS (1979) and CENTENo (1986) and, in a certain way, this work may be considered as a generalization of their results. Although this criterion does not by any means have to minimize the (analytically uncalculable) ruin probability, it is a good criterion if one wishes to give analytical results.

Surplus and stop loss treaties are not considered in this paper. WATERS (1983), derives sufficient conditions for the adjustment coefficient to be uni-model, for stop loss reinsurance. 
For each $i=1,2, \ldots, n$, let $a_{i}$, be the decision variable representing the quota-share retention on risk $i ; M_{i}$, the decision variable representing the excess of loss retention limit on risk $i ; Y_{i}=\sum_{j=0}^{N_{i}} X_{i j}$, with $X_{i 0} \equiv 0$, the insurer's aggregate gross (of reinsurance) claims on risk $i$, in some fixed time interval, where $N_{i}$ is the number of claims and $\left\{X_{i j}\right\}_{j=1, \ldots, N_{i}}$ are the individual claims; $P_{i}$ the insurer's gross (of expenses and reinsurance) premium income with respect to risk $i$ and $e_{i} P_{i}$ the amount used to cover the insurer's expenses with respect to the same risk.

After a combination of a quota-share with an excess of loss treaty the insurer will retain, from risk $i, Y_{i}\left(a_{i}, M_{i}\right)=\sum_{j=1}^{N_{i}} \min \left\{a_{i} X_{i j}, M_{i}\right\},(i=1, \ldots, n)$.

The choice of uniform $a_{1}=\ldots=a_{n}$ and $M_{1}=\ldots=M_{n}$, which is generally made in practice, has been dealt with in CENTENO (1986). In this paper, therefore, retention limits which can, for instance, be set differently for portfolios of different classes of business are also dealt with.

Let $P_{t i}\left(a_{i}, M_{i}\right)$ be the total reinsurance premium paid by the insurer, in respect to risk $i$ (it is, naturally, the summation of the quota-share and excess of loss reinsuance premiums).

The problem which is to be solved is, then,

$\begin{array}{ll}\text { Maximize } & R(\underline{a}, \underline{M}) \\ \text { sub. to: } & 0 \leq a_{i} \leq 1 \\ & M_{i} \geq 0 \\ & (i=1,2, \ldots, n),\end{array}$

where $R(\underline{a}, \underline{M})$, is the adjustment coefficient, defined, as it is known, as the unique positive root of

$$
E\left[\exp \left\{R \sum_{i=1}^{n} Y_{i}\left(a_{i}, M_{i}\right)-R \sum_{i=1}^{n}\left[P_{i}\left(1-e_{i}\right)-P_{t i}\left(a_{i}, M_{i}\right)\right]\right\}\right]=1 .
$$

Note that $R(\underline{a}, \underline{M})$ is the adjustment coefficient (see Beard, Pentikainen and Pesonen (1984), p. 363) after taking account of the reinsurance arrangement.

\section{ASSUMPTIONS AND PREMIMINARIES}

$A_{1}: \quad Y_{i}(i=1,2, \ldots, n)$ are independent random variables;

For each $i(i=1,2, \ldots, n)$ :

$A_{2}: \quad N_{i}(i=1,2, \ldots, n)$ is a Poisson random variable with parameter $\lambda_{i}$; 
$A_{3}:\left\{X_{i j}\right\}_{j=1,2, \ldots, N_{i}}$ are i.i.d. non-negative random variables, independent of $N_{i}$, and with common distribution function $F_{i}$ such that

$\left\{\begin{aligned} F_{i}(x) & =0, & & x \leq x_{i 0} \\ 0<F_{i}(x) & <1, & & x>x_{i 0},\end{aligned}\right.$

for some $x_{i 0} \geq 0$;

$A_{4}: \frac{d}{d x} F_{i}(x)$ exists and it is continuous everywhere;

$A_{5}$ : The m.g.f. of the random variables $X_{i j}$, exists in the $\left(-\infty, Q_{i}\right]$ interval, for $0<Q_{i} \leq+\infty$ and

$$
\lim _{t_{i} \rightarrow Q_{i}} E\left[e^{t_{i} X_{i j}}\right]=+\infty
$$

$A_{6}$ : The quota-share reinsurance premium is

$\left(1-a_{i}\right) P_{i}-c_{i}\left(1-a_{i}\right) P_{i}=\left(1-c_{i}\right)\left(1-a_{i}\right) P_{i}$,

where $c_{i}\left(1-a_{i}\right) P_{i}, 0<c_{i}<1$, is the habitual commission paid by the quota-share reinsurer;

$A_{7}$ : The excess of loss reinsurance premium, which we denote $P_{i}\left(a_{i}, M_{i}\right)$, is calculated according to the expected value principle, i.e.,

$$
P_{i}\left(a_{i}, M_{i}\right)=\left(1+\alpha_{i}\right) \lambda_{i} \int_{M_{i} / a_{i}}^{+\infty}\left(a_{i} x-M_{i}\right) d F_{i}(x)
$$

with $\alpha_{i}>0$.

$A_{8}: \quad e_{i}>c_{i}$;

$A_{9}:\left(1-c_{i}\right) P_{i}-\lambda_{i} E\left[X_{i}\right]>0$, where $E\left[X_{i}\right]$ denotes the expected value of $X_{i j}$, $j=1,2, \ldots, N_{i}$

$A_{10}:\left(1-e_{i}\right) P_{i}<\left(1+\alpha_{i}\right) \lambda_{i} E\left[X_{i}\right]$;

Finally, we assume that

$A_{11}: \sum_{i=1}^{n}\left[\left(1-e_{i}\right) P_{i}-\lambda_{i} E\left[X_{i}\right]\right]>0$.

From $A_{2}$ and $A_{3}$ it follows that $Y_{i}$ and $Y_{i}\left(a_{i}, M_{i}\right)$ have compound Poisson distributions. From $A_{6}$ and $A_{7}$ we can say that

(2) $P_{t i}\left(a_{i}, M_{i}\right)=\left(1-c_{i}\right)\left(1-a_{i}\right) P_{i}+\left(1+\alpha_{i}\right) \lambda_{i} \int_{M_{i} / a_{i}}^{+\infty}\left(a_{i} x-M_{i}\right) d F_{i}(x)$. 
Assumption $A_{8}$ is somewhat restrictive, but without it the insurer could reinsure the whole risk through a quota-share arrangement with a certain profit. The same applies to $A_{10}$, but with respect to the excess of loss reinsurance treaty. $A_{9}$ implies that the loading on the quota-share reinsurance premium is positive. At last, $A_{11}$ assures the existence of a margin, necessary to cover eventual deviations from the expected losses, and also to pay the reinsurance costs.

Under assumptions $A_{1}, A_{2}$ and $A_{3}, R(\underline{a}, \underline{M})$ is the only positive root of

$$
G(R ; \underline{a}, \underline{M})=0 \text {, }
$$

where

(4) $\quad G(R ; \underline{a}, \underline{M})=\sum_{i=1}^{n} \lambda_{i}\left[\int_{0}^{M_{i} / a_{i}} e^{R a_{i} x} d F_{i}(x)+e^{R M_{i}}\left[1-F_{i}\left(M_{i} / a_{i}\right)\right]-1\right]-$

$$
-R \sum_{i=1}^{n}\left[\left(1-e_{i}\right) P_{i}-P_{t i}\left(a_{i}, M_{i}\right)\right]
$$

(See Beard, Pentikainen and Pesonen (1984), p. 363, for the equivalence of (4) and (1).) Let $E[W(\underline{a}, \underline{M})]$ denote the insurer's expected net profit, after reinsurance and expenses, $\overline{\text { i.e., }}$

$$
\begin{aligned}
E[W(\underline{a}, \underline{M})= & \sum_{i=1}^{n}\left[\left(c_{i}-e_{i}\right) P_{i}+a_{i}\left[\left(1-c_{i}\right) P_{i}-\lambda_{i} E\left[X_{i}\right]\right]-\right. \\
& \left.-\lambda_{i} \alpha_{i} \int_{M_{i} / a_{i}}^{+\infty}\left(a_{i} x-M_{i}\right) d F_{i}(x)\right]
\end{aligned}
$$

and let us define

$$
\Upsilon=\left\{(\underline{a}, \underline{M}): 0 \leq a_{i} \leq 1, \quad M_{i} \geq 0 \text { and } E[W(\underline{a}, \underline{M})]>0\right\},
$$

and

$\Gamma=\left\{\underline{a}: 0 \leq a_{i} \leq 1, i=1,2, \ldots, n\right.$ and there exists at least one $\underline{M}$ such that $E[W(\underline{a}, \underline{M})]>0\}$.

Since

$$
\frac{\partial}{\partial M_{i}} E[W(\underline{a}, \underline{M})]=\lambda_{i} \alpha_{i}\left(1-F_{i}\left(M_{i} / a_{i}\right)\right)
$$

is non-negative, we can say that for fixed $\underline{a}$, the expected net profit will be maximum when $M_{i}=+\infty(i=1,2, \ldots, n)$. Hence it is possible to specify $\Gamma$ as being

$$
\Gamma=\left\{\underline{a}: \sum_{i=1}^{n}\left[\left(c_{i}-e_{i}\right) P_{i}+a_{i}\left[\left(1-c_{i}\right) P_{i}-\lambda_{i} E\left[X_{i}\right]\right]\right]>0\right\}
$$


Let us denote $\frac{\partial}{\partial R} G(R ; \underline{a}, \underline{M})$ by $D(R ; \underline{a}, \underline{M})$ so that

(7) $D(R ; \underline{a}, \underline{M})=\sum_{i=1}^{n} \lambda_{i}\left[\int_{0}^{M_{i} / a_{i}} a_{i} x e^{R a_{i} x} d F_{i}(x)+M_{i} e^{R M_{i}}\left(1-F_{i}\left(M_{i} / a_{i}\right)\right)\right]-$ $-\sum_{i=1}^{n}\left[\left(1-e_{i}\right) P_{i}-P_{t i}\left(a_{i}, M_{i}\right)\right]$

with $G(R ; \underline{a}, \underline{M})$ defined by (4).

The following lemma discusses the existence of the adjustment coefficient.

\section{Lemma 1:}

(i) $R(\underline{a}, \underline{M})$ exists, if and only if $(\underline{a}, \underline{M}) \in \Upsilon$;

(ii) For any $(\underline{a}, \underline{M}) \in \Upsilon, D(R ; \underline{a}, \underline{M})$ is positive at $R=R(\underline{a}, \underline{M})$.

\section{Proof:}

(i) By $A_{5}$, it is clear that for fixed $(\underline{a}, \underline{M}), G$ is defined for all $R \in(-\infty, Q)$,

where

$$
Q=\min \left\{\zeta_{i}\right\}
$$

and

$$
\begin{gathered}
\zeta_{i}= \begin{cases}+\infty, & \text { if } \quad M_{i} / a_{i}<+\infty \\
\frac{Q_{i}}{a_{i}}, & \text { if } \quad M_{i} / a_{i}=+\infty\end{cases} \\
(i=1,2, \ldots, n) .
\end{gathered}
$$

The first aspect to be considered, is that $R=0$ is a trivial solution of equation (3);

Secondly, we have that

$$
\frac{\partial^{2}}{\partial R^{2}} G(R ; \underline{a}, \underline{M})=\sum_{i=1}^{n} \lambda_{i}\left[\int_{0}^{M_{i} / a_{i}}\left(a_{i} x\right)^{2} e^{R a_{i} x} d F_{i}(x)+M_{i}^{2} e^{R M_{i}}\left(1-F_{i}\left(M_{i} / a_{i}\right)\right)\right],
$$

it is non-negative, $\forall(\underline{a}, \underline{M})$, which means that $G(R ; \underline{a}, \underline{M})$ is a convex function of $R$; 
Third,

$$
\begin{aligned}
\lim _{R \rightarrow Q} G(R ; \underline{a}, \underline{M})= & \sum_{i=1}^{n} \lim _{R \rightarrow Q}\left\{\lambda_{i} \int_{0}^{M_{i} / a_{i}}\left[e^{R a_{i} x}-R\left(1+\alpha_{i}\right) a_{i} x\right] d F_{i}(x)+\right. \\
& +\lambda_{i}\left[e^{R M_{i}}-R\left(1+\alpha_{i}\right) M_{i}\right]\left(1-F_{i}\left(M_{i} / a_{i}\right)\right)-\lambda_{i}- \\
& \left.-R\left[\left(c_{i}-e_{i}\right) P_{i}+a_{i}\left[\left(1-c_{i}\right) P_{i}-\left(1+\alpha_{i}\right) \lambda_{i} E\left[X_{i}\right]\right]\right]\right\} \\
= & +\infty
\end{aligned}
$$

by assumptions $A_{8}$ and $A_{9}$.

Hence, as $G(R ; \underline{a}, \underline{M})$ equals zero when $R$ is null, $G(R ; \underline{a}, \underline{M})$ is a convex function of $R$, and $G(\bar{R} ; \underline{a}, \underline{M})$ tends to infinity when $R$ tends to $Q$, then, it will only exist such an $R=\bar{R}(\underline{a}, \underline{M})>0$ which turns $G(R ; \underline{a}, \underline{M})$ to be null again, if and only if,

$$
\left.\frac{\partial}{\partial R} G(R ; \underline{a}, \underline{M})\right|_{R=0}<0 .
$$

To finish the prof, we only have to notice that

$$
\left.\frac{\partial}{\partial R} G(R ; \underline{a}, \underline{M})\right|_{R=0}<0 \Leftrightarrow E[W(\underline{a}, \underline{M})]>0 .
$$

(ii) Immediate, given the proof of (i).

The following lemma will be useful to the solution to our problem.

Lemma 2: For any $\underline{a} \in \Gamma$ there exists a unique $(\underline{a}, \underline{M}) \in \Upsilon$, let it be $(\underline{a}, \underline{\hat{M}})$, such that

$$
R(\underline{a}, \underline{\hat{M}})=\frac{\ln \left(1+\alpha_{i}\right)}{\hat{M}_{i}}, \quad i=1,2, \ldots, n
$$

Proof: Let us consider the set of points $\underline{M}$ such that

$$
\frac{\ln \left(1+\alpha_{1}\right)}{M_{1}}=\frac{\ln \left(1+\alpha_{2}\right)}{M_{2}}=\ldots=\frac{\ln \left(1+\alpha_{n}\right)}{M_{n}}=\frac{1}{\tilde{M}}, \quad \tilde{M}>0
$$

and let us define

$$
H(\underline{a}, \tilde{M})=\tilde{M} G\left(\frac{1}{\tilde{M}} ; \underline{a}, \tilde{M} \ln \left(1+\alpha_{1}\right), \ldots, \tilde{M} \ln \left(1+\alpha_{n}\right)\right),
$$


which is to say

$$
\begin{aligned}
H(\underline{a}, \tilde{M})= & \sum_{i=1}^{n}\left\{\lambda_{i} \int_{0}^{\tilde{\mu} \frac{\ln \left(1+\alpha_{i}\right)}{a_{i}}} \tilde{M} e^{\frac{1}{\tilde{M}} a_{i} x} d F_{i}(x)+\right. \\
& +\lambda_{i} \tilde{M}\left(1+\alpha_{i}\right)\left[1-F_{i}\left(\tilde{M} \frac{\ln \left(1+\alpha_{i}\right)}{a_{i}}\right)\right]-\tilde{M} \lambda_{i}- \\
& -\left[\left(1-e_{i}\right) P_{i}-\left(1-c_{i}\right)\left(1-a_{i}\right) P_{i}\right]+ \\
& \left.+\left(1+\alpha_{i}\right) \lambda_{i} \int_{\tilde{M} \frac{\ln \left(1+\alpha_{i}\right)}{a_{i}}}^{+\infty}\left(a_{i} x-\tilde{M} \ln \left(1+\alpha_{i}\right)\right) d F_{i}(x)\right\}
\end{aligned}
$$

Then

$10 \lim _{\tilde{M} \rightarrow 0+} H(\underline{a}, \tilde{M})=\sum_{i=1}^{n}-\left[\left(1-e_{i}\right) P_{i}-\left(1-c_{i}\right)\left(1-a_{i}\right) P_{i}-\right.$

$$
\left.-\left(1+\alpha_{i}\right) \lambda_{i} a_{i} E\left[X_{i}\right]\right]>0,
$$

using $A_{8}$ and $A_{10}$;

2) $\lim _{\tilde{M} \rightarrow+\infty} H(\underline{a}, \tilde{M})=-\sum_{i=1}^{n}\left\{\left[\left(c_{i}-e_{i}\right) P_{i}+a_{i}\left[\left(1-c_{i}\right) P_{i}-\right.\right.\right.$

$$
\left.\left.-\lambda_{i} E\left[X_{i}\right]\right]\right\}<0
$$

3) Differentiating $H(\underline{a}, \tilde{M})$ twice with respect to $\tilde{M}$ we obtain (see, for example, COURANT and JoHn (1974), p. 77)

$$
\frac{\partial^{2}}{\partial \tilde{M}^{2}} H(\underline{a}, \tilde{M})=\sum_{i=1}^{n} \lambda_{i} \int_{0}^{\tilde{M} \frac{\ln \left(1+\alpha_{i}\right)}{a_{i}}} e^{\frac{1}{\tilde{M}} a_{i} x} \frac{\left(a_{i} x\right)^{2}}{\tilde{M}^{3}} d F_{i}(x) \geq 0 .
$$

Hence, for each $\underline{a} \in \Gamma$ there exists a unique positive $\tilde{M}=\tilde{M}(\underline{a})$ such that $H(\underline{a}, \tilde{M})=0$ and it is clear from the definition of $H(\underline{a}, \tilde{M})$ that

$$
G\left(\frac{1}{\tilde{M}} ; \underline{a}, \underline{\hat{M}}\right)=0
$$

where

$\underline{\hat{M}}=\left(\hat{M}_{1}, \hat{M}_{2}, \ldots, \hat{M}_{n}\right)=\left(\tilde{M} \ln \left(1+\alpha_{1}\right), \tilde{M} \ln \left(1+\alpha_{2}\right), \ldots, \tilde{M} \ln \left(1+\alpha_{n}\right)\right) \diamond$

This lemma implies that if we define

(8) $\hat{G}(\hat{R} ; \underline{a})=G\left(\hat{R} ; \underline{a}, \hat{R}^{-1} \ln \left(1+\alpha_{1}\right), \hat{R}^{-1} \ln \left(1+\alpha_{2}\right), \ldots, \hat{R}^{-1} \ln \left(1+\alpha_{n}\right)\right)$, 
then $\hat{G}(\hat{R} ; \underline{a})$ has a unique positive root for each $a \in \Gamma$. Let us denote it $\hat{R}(\underline{a})$. It can be proved, using the Implicit Function Theorem (see for example Courant and JoHn (1974), pp. 221-223), Part (2) of Lemma 1 and $A_{4}$, that $R(a, \underline{M})$, for $(\underline{a}, \underline{M}) \in \Upsilon$, and $\hat{R}(\underline{a})$, for $\underline{a} \in \Gamma$, are twice differentiable.

\section{THE SOLUTION TO THE PROBLEM}

The following result provides the solution to our problem.

\section{Result 1:}

(i) For a fixed value $\underline{a} \in \Gamma$, with $a_{i} \neq 0, \forall i=1,2, \ldots, n, R(\underline{a}, \underline{M})$ is a unimodal function of $\underline{M}$, and for any $\underline{a} \in \Gamma$ its maximum value is $\hat{R}(\underline{a})$.

(ii) $\hat{R}(\underline{a})$ is a unimodal function of $\underline{a}$, for $\underline{a} \in \Gamma$ and, at the point where it attains its maximum:

a) $a_{i}=1$ if and only if $\frac{\partial}{\partial a_{i}} \hat{R}(\underline{a})\left(a_{i}=1\right) \geq 0$,

or

b) $a_{i}$ is such that $\frac{\partial}{\partial a_{i}} \hat{R}(\underline{a})=0$, if and only if

$$
\frac{\partial^{\prime}}{\partial a_{i}} \hat{R}(\underline{a})\left(a_{i}=1\right) \leq 0, i=1,2, \ldots, n .
$$

\section{Proof:}

(i) The equation defining $R(\underline{a}, \underline{M})$ for all $(\underline{a}, \underline{M}) \in \Gamma$ is

$$
G(R ; \underline{a}, \underline{M})=0,
$$

with $G(R ; \underline{a}, \underline{M})$ given by (4). Differentiating (9) with respect to $M_{i}$ it can be seen that $\frac{\partial}{\partial M_{i}} R(\underline{a}, \underline{M})=0$ if and only if (using the Implicit Function Theorem)

$$
\operatorname{Re}^{R M_{i}}\left(1-F_{i}\left(M_{i} / a_{i}\right)\right)=R\left(1+\alpha_{i}\right)\left(1-F_{i}\left(M_{i} / a_{i}\right)\right) .
$$

So, using Lemma 2 we can say that for a fixed value of $a \in \Gamma$, with $a_{i} \neq 0$, $\forall i=1,2, \ldots, n$, the only turning point of $R(\underline{a}, \underline{M})$ is such that

$$
M_{i}=R^{-1} \ln \left(1+\alpha_{i}\right), \quad i=1,2, \ldots, n .
$$


Differentiating (9) twice with respect to $M_{i}$ (using again the Implicit Function Theorem and (10)) we get

$$
\left.\frac{\partial^{2}}{\partial M_{i}^{2}} R(\underline{a}, \underline{M})\right|_{M_{i}=R^{-1} \ln \left(1+\alpha_{i}\right)}=-\left.\frac{\lambda_{i} R^{2} e^{R M_{i}}\left[1-F_{i}\left(M_{i} / a_{i}\right)\right]}{D(R ; \underline{a}, \underline{M})}\right|_{M_{i}=R^{-1} \ln \left(1+\alpha_{i}\right)}
$$

with $D(R ; \underline{a}, \underline{M})$ given by (7). We can see that each side of equation (11) is negative since $\bar{D}(R ; \underline{a}, \underline{M})$ is positive by Lemma 1 (ii).

On the other hand,

$$
\left.\frac{\partial^{2}}{\partial M_{i} \partial M_{j}} R(\underline{a}, \underline{M})\right|_{\substack{M_{i}=R^{-1} \ln \left(1+\alpha_{i}\right) \\ M_{j}=R^{-1} \ln \left(1+\alpha_{j}\right)}}=0, \quad j \neq i .
$$

Hence we can conclude that for a fixed value $\underline{a} \in \Gamma$ with $a_{i} \neq 0$, $\forall i=1,2, \ldots, n, R(\underline{a}, \underline{M})$ is a unimodal function of $\underline{M}$.

If $\underline{a} \in \Gamma$ and $a_{k}=0$ for some $k=1,2, \ldots, n$, then of course any value for the excess of loss retention limit of risk $k$, including $M_{k}=R^{-1} \ln \left(1+\alpha_{k}\right)$, will provide the same value for the adjustment coefficient.

Then the maximum of $R(\underline{a}, \underline{M})$ is attained at the point $(\underline{a}, \underline{M})$ which is the unique point satisfying $\bar{G}(R ; \underline{a}, \underline{M})=0$ and $M_{i}=R^{-1} \ln \left(1+\alpha_{i}\right)$, $i=1,2, \ldots, n$, i.e., for a fixed $\underline{a} \in I$, the maximum of $R(\underline{a}, \underline{M})$ is $\hat{R}$ where $\hat{R}=\hat{R}(\underline{a})$ is the only positive root of $\hat{G}(\hat{R} ; \underline{a})=0$, with $\hat{G}(\hat{R} ; \underline{a})$ given by (8).

(ii) Differentiating

$$
\hat{G}(\hat{R} ; \underline{a})=0
$$

with respect to $a_{i}$ we obtain

$$
\begin{aligned}
\frac{\partial}{\partial a_{i}} \hat{R}(\underline{a})= & \frac{\hat{R}}{\hat{D}(\hat{R} ; \underline{a})}\left[\left(1-c_{i}\right) P_{i}-\left(1+\alpha_{i}\right) \lambda_{i} \int_{\frac{\ln \left(1+\alpha_{i}\right)}{\hat{R} a_{i}}}^{+\infty} x d F_{i}(x)-\right. \\
& \left.-\lambda_{i} \int_{0}^{-\frac{\ln \left(1+\alpha_{i}\right)}{\hat{R} a_{i}}} x e^{\hat{R} a_{i} x} d F_{i}(x)\right]
\end{aligned}
$$

where

$$
\hat{D}(\hat{R} ; \underline{a})=D\left(\hat{R} ; \underline{a}, \hat{R}^{-1} \ln \left(1+\alpha_{1}\right), \hat{R}^{-1} \ln \left(1+\alpha_{2}\right), \ldots, \hat{R}^{-1} \ln \left(1+\alpha_{n}\right)\right) .
$$

So,

$$
\frac{\partial}{\partial a_{i}} \hat{R}(\underline{a})=0
$$

if and only if

$$
\left(1-c_{i}\right) P_{i}=\lambda_{i} \int_{0}^{\frac{\ln \left(1+\alpha_{i}\right)}{\hat{R} a_{i}}} x e^{\hat{R} a_{i} x} d F_{i}(x)+\left(1+\alpha_{i}\right) \lambda_{i} \int_{\frac{\ln \left(1+\alpha_{i}\right)}{\hat{R} a_{i}}}^{+\infty} x d F_{i}(x)
$$


Differentiating (13) with respect to $a_{i}$, and using (14), we obtain

$$
\left.\frac{\partial^{2}}{\partial a_{i}^{2}} \hat{R}(\underline{a})\right|_{\frac{\partial}{\partial a_{i}} \hat{R}(\underline{a})=0}=-\left.\frac{\lambda_{i} \int_{0}^{\frac{\ln \left(1+\alpha_{i}\right)}{\hat{R} a_{i}}} \hat{R}^{2} x^{2} e^{\hat{R} a_{i} x} d F_{i}(x)}{\hat{D}(\hat{R} ; \underline{a})}\right|_{\frac{\partial}{\partial a_{i}} \hat{R}(\underline{a})=0}
$$

and

$$
\left[\frac{\partial^{2}}{\partial a_{i} a_{j}} \hat{R}(\underline{a})\right]\left(\frac{\partial}{\partial a_{i}} \hat{R}(\underline{a})=0, \frac{\partial}{\partial a_{j}} \hat{R}(\underline{a})=0\right)=0 \text {, if } i \neq j .
$$

This implies that there exists at most a point $\underline{a} \in \Gamma$ such that (14) holds for $i=1,2, \ldots, n$.

Noticing that

$$
\lim _{a_{i} \rightarrow 0+} \frac{\partial}{\partial a_{i}} \hat{R}(\underline{a})=\left[\left(1-c_{i}\right) P_{i}-\lambda_{i} E\left[X_{i}\right]\right] \lim _{a_{i} \rightarrow 0+} \frac{\hat{R}}{\hat{D}(\hat{R} ; \underline{a})},
$$

with $\underline{a} \in \Gamma$, is positive by $A_{9}$ and Lemma 1 (ii), the proof is finished.

To summarize, we can now conclude that the optimum programme of reinsurance, when a company is to reinsure $n$ independent risks by a combination of the quota share an excess of loss forms of reinsurance, is the point $(\underline{a}, \underline{M})$ which fulfils the following set of conditions:

$$
\begin{aligned}
& \rightarrow M_{i}=\frac{\ln \left(1+\alpha_{i}\right)}{R}, \quad(i=1,2, \ldots, n) \\
& \rightarrow a_{i}: \quad\left(1-c_{i}\right) P_{i}=\lambda_{i} \int_{0}^{\frac{\ln \left(1+\alpha_{i}\right)}{R a_{i}}} x e^{R a_{i} x} d F_{i}(x)+\left(1+\alpha_{i}\right) \lambda_{i} \int_{\frac{\ln \left(1+\alpha_{i}\right)}{R a_{i}}}^{+\infty} x d F_{i}(x) \\
& \quad \text { if }\left[\frac{\partial R}{\partial a_{i}}\right]<0 \text { when } a_{i}=1 \\
& \rightarrow \text { or } \quad a_{i}=1, \quad \text { if }\left[\frac{\partial R}{\partial a_{i}}\right] \geq 0 \text { when } a_{i}=1 \\
& \quad(i=1,2, \ldots, n) \\
& \rightarrow G(R ; \underline{a}, \underline{M})=0
\end{aligned}
$$

Corollary 1 : If $\left(1-c_{i}\right) P_{i} \geq \lambda_{i}\left(1+\alpha_{i}\right) E\left[X_{i}\right]$ for some $i(i=1,2, \ldots, n)$, then the optimal arrangement is such that $a_{i}=1$. 
Proof: We only have to notice that in this case

$$
\begin{gathered}
\left(1-c_{i}\right) P_{i}-\lambda_{i} \int_{0}^{R^{-1} \ln \left(1+\alpha_{i}\right)} x e^{R x} d F_{i}(x)-\left(1+\alpha_{i}\right) \lambda_{i} \int_{R^{-1} \ln \left(1+\alpha_{i}\right)}^{\infty} x d F_{i}(x) \geq \\
\geq \lambda_{i} \int_{0}^{R^{-1} \ln \left(1+\alpha_{i}\right)} x\left(1+\alpha_{i}-e^{R x}\right) \geq 0 . \quad \diamond
\end{gathered}
$$

Note that we can regard the quota-share reinsurance premium for risk $i$ (see $A_{6}$ ) as being calculated using the expected value principle with loading factor $\hat{\alpha}_{i}$, where

$$
\hat{\alpha}_{i}=\left[\left(1-c_{i}\right)-\lambda_{i} E\left[X_{i}\right]\right] /\left[\lambda_{i} E\left[X_{i}\right]\right] .
$$

Then, Corollary 1 implies that if $\hat{\alpha}_{i} \geq \alpha_{i}$, i.e. if quota-share is, in the obvious sence more expensive than excess of loss reinsurance, then excess of loss reinsurance is optimal. Excess of loss reinsurance was already proved to be the optimal form of reinsurance (see GERBER 1979), p. 129), in the sence that it maximizes the adjustment coefficient, under the assumption that the loading coefficient is the same for the insurer and the reinsurer (which is not the case in our paper).

When the number of risks, $n$, is greater than one, the solution found for the problem, may not be the solution that we would obtain if the risks were considered separately. In other words, if we regard as optimal a set of retention limits that maximizes the adjustment coefficient, then what is optimal when each risk is considered individually may not be optimal when the risks are considered together, as we will see next.

In the result that follows, $R\left(a_{i}, M_{i}\right)(i=1,2, \ldots, n)$ is, for fixed $\left(a_{i}, M_{i}\right)$, the adjustment coefficient associated to risk $i$, when this is considered on its own, defined as the unique positive root of

$$
G_{i}\left(R_{i} ; a_{i}, M_{i}\right)=0
$$

where

$$
\begin{aligned}
G_{i}\left(R_{i} ; a_{i}, M_{i}\right)= & \lambda_{i}\left[\int_{0}^{M_{i} / a_{i}} e^{R_{i} a_{i} x} d F_{i}(x)+e^{R_{i} M_{i}}\left[1-F_{i}\left(M_{i} / a_{i}\right)\right]-1\right]- \\
& -R_{i}\left[\left(1-e_{i}\right) P_{i}-P_{t i}\left(a_{i}, M_{i}\right)\right]
\end{aligned}
$$

if such a root exists, or zero otherwise. ${ }^{1}$

Result 2: For fixed $(\underline{a}, \underline{M}) \in \Upsilon$ we have

$$
\min _{i=1, \ldots, n}\left\{R_{i}\left(a_{i}, M_{i}\right)\right\} \leq R(\underline{a}, \underline{M}) \leq \max _{i=1, \ldots, n}\left\{R_{i}\left(a_{i}, M_{i}\right)\right\}
$$

1 The need to redefine $R_{i}\left(a_{i}, M_{i}\right)$ comes from the fact that $E[W(\underline{a}, \underline{M})]>0$ does not imply that $E\left[W_{i}\left(a_{i}, M_{i}\right)\right]>0$, for all $i=1,2, \ldots, n$. 


\section{Proof :}

Let

$$
\min _{i=1, \ldots, n}\left\{R_{i}\left(a_{i}, M_{i}\right)\right\}=R_{k}\left(a_{k}, M_{k}\right)
$$

and

$$
\max _{i=1, \ldots, n}\left\{R_{i}\left(a_{i}, M_{i}\right)\right\}=R_{l}\left(a_{l}, M_{l}\right) .
$$

Then, considering the definition of $R_{i}\left(a_{i}, M_{i}\right), \forall i=1,2, \ldots, n$, we have that

$$
0 \leq R_{k}\left(a_{k}, M_{k}\right) \leq R_{l}\left(a_{l}, M_{l}\right)
$$

and, on the other hand, having in mind the proof of Lemma 1, we know that

$$
\left\{\begin{array}{rrr}
G_{i}\left(R_{i} ; a_{i}, M_{i}\right)<0 & \text { if } & 0<R_{i}<R_{i}\left(a_{i}, M_{i}\right) \\
G_{i}\left(R_{i} ; a_{i}, M_{i}\right)>0 & \text { if } & R_{i}>R_{i}\left(a_{i}, M_{i}\right)
\end{array}\right.
$$

for $i=1,2, \ldots, n$.

From (19) and attending to (17) and (18) we have that

$$
\sum_{i=1}^{n} G_{i}\left(R_{k}\left(a_{k}, M_{k}\right) ; a_{i}, M_{i}\right) \leq 0,
$$

being zero if and only if $R_{k}\left(a_{k}, M_{k}\right)=R_{l}\left(a_{l}, M_{l}\right)$. Similiary

$$
\sum_{i=1}^{n} G_{i}\left(R_{l}\left(a_{l}, M_{l}\right) ; a_{i}, M_{i}\right) \geq 0,
$$

being zero if and only if $R_{k}\left(a_{k}, M_{k}\right)=R_{l}\left(a_{l}, M_{l}\right)$ :

Then the result follows immediately, since $R(\underline{a}, \underline{M})$ for $(\underline{a}, \underline{M}) \in \Upsilon$ is the unique positive root of

$$
\sum_{i=1}^{n} G_{i}\left(R ; a_{i}, M_{i}\right)=0 \diamond
$$

Corollary 2: If $R_{i}\left(a_{i}, M_{i}\right)$ achieves its maximum value at $\left(a_{i}, M_{i}\right)=\left(\hat{a}_{i}, \hat{M}_{i}\right)$, $i=1,2, \ldots, n$, and if $R(\underline{a}, \underline{M})$ achieves its maximum value at $(\underline{a}, \underline{M})=(\underline{\hat{a}}, \underline{\hat{M}})$, then

$$
\min _{i=1, \ldots, n}\left\{R_{i}\left(\hat{a}_{i}, \hat{M}_{i}\right)\right\} \leq R(\underline{\hat{\hat{a}}}, \underline{\hat{\hat{M}}}) \leq \max _{i=1, \ldots, n}\left\{R_{i}\left(\hat{a}_{i}, \hat{M}_{i}\right)\right\}
$$


Proof: Attending to Result 2, to the definition of $(\underline{\hat{\hat{a}}}, \underline{\hat{\hat{M}}})$ and to the definition of $\left(\hat{a}_{i}, \hat{M}_{i}\right), i=1,2, \ldots, n$, then

$$
\min _{i=1, \ldots, n}\left\{R_{i}\left(\hat{a}_{i}, \hat{M}_{i}\right)\right\} \leq R(\underline{\hat{a}}, \underline{\hat{M}}) \leq R(\underline{\hat{\hat{a}}}, \underline{\hat{\hat{M}}}),
$$

and

$$
R(\underline{\hat{\hat{a}}}, \underline{\hat{\hat{M}}}) \leq \max _{i=1, \ldots, n}\left\{R_{i}\left(\hat{\hat{a}}_{i}, \hat{\hat{M}}_{i}\right)\right\} \leq \max _{i=1, \ldots, n}\left\{R_{i}\left(\hat{a}_{i}, \hat{M}_{i}\right)\right\}
$$

which finishes the proof.

\section{EXAMPLE}

Let $n=2$ and

$$
G_{1}(x)= \begin{cases}0, & \text { if } x \leq 0 \\ 1-\frac{1}{4} e^{-\frac{1}{4} x}(x+4), & \text { if } x>0\end{cases}
$$

which corresponds to a $\gamma\left(2, \frac{1}{4}\right)$, and

$$
G_{2}(x)= \begin{cases}0 & \text { if } x \leq 1 \\ 1-e^{-3(x-1)}, & \text { if } x>1\end{cases}
$$

which is an exponencial.

Let $\lambda_{1}=2, \lambda_{2}=10, P_{1}=27, P_{2}=23.5, e_{1}=e_{2}=.35, U_{1}=30$ and $U_{2}=15$. The expected profit, before any reinsurance arrangement takes place, is 3.491(6) (1.55 from risk 1 and 1.941(6) from risk 2), $R$ is .02849 and, therefore, the upper bound given by Lundberg's inequality for the ruin probability, is 0.2774 . Considering the two risks separately the adjustment coefficients are $R_{1}=0.01487$ and $R_{2}=0.1864$, giving then upper bounds for the ruin probabilities of 0.6401 and 0.0610 , for risks 1 and 2 respectively.

The optimal reinsurance programme was calculated assuming different values for $\alpha_{1}$ and setting $\alpha_{2}=.3, c_{1}=c_{2}=.25$. The results can be seen on Table 1. Analysing Table 1, the main aspect that seems evident is that, as long as $\alpha_{1}$ increases, a similar evolution is presented by ratio $M_{1} / a_{1}$, that is to say, the excess of loss form of reinsurance becomes less and less attractive.

Table 2 gives the same kind of information as Table 1, when treating the two risks separately. Note that $R_{1}<R<R_{2}$. One way of explaining this occurrence may be the following: when the reinsurance problem is solved taking the risks together, there is a sort of a transfer of part of the income produced for the "less dangerous" (and, therefore "less needed" of reinsurance) risks, to 
TABLE 1

\begin{tabular}{|c|c|c|c|c|}
\hline$\alpha_{1}$ & $\begin{array}{c}\text { Optimal } \\
\text { Retentions }\end{array}$ & $\begin{array}{l}\text { Expected } \\
\text { Net Profit }\end{array}$ & $\begin{array}{l}\text { Adjustment } \\
\text { Coefficient }\end{array}$ & $\begin{array}{l}\text { Upper Bound } \\
\text { by Lundberg's } \\
\text { Inequality }\end{array}$ \\
\hline 0.3 & $\begin{aligned} a_{1} & =00.77 \\
a_{2} & =01.00 \\
M_{1} & =06.10 \\
M_{2} & =06.10\end{aligned}$ & 1.4986 & 0.04300 & 0.1444 \\
\hline 0.4 & $\begin{aligned} a_{1} & =00.57 \\
a_{2} & =01.00 \\
M_{1} & =08.59 \\
M_{2} & =06.69\end{aligned}$ & 1.4177 & 0.03919 & 0.1714 \\
\hline 0.5 & $\begin{aligned} a_{1} & =00.53 \\
a_{2} & =01.00 \\
M_{1} & =10.59 \\
M_{2} & =06.86\end{aligned}$ & 1.3946 & 0.03827 & 0.1787 \\
\hline 0.6 & $\begin{aligned} a_{1} & =00.52 \\
a_{2} & =01.00 \\
M_{1} & =12.39 \\
M_{2} & =06.92\end{aligned}$ & 1.3846 & 0.03794 & 0.1814 \\
\hline
\end{tabular}

TABLE 2

\begin{tabular}{|c|c|c|c|c|}
\hline$\alpha_{1}$ & $\begin{array}{c}\text { Optimal } \\
\text { Retentions }\end{array}$ & $\begin{array}{l}\text { Expected } \\
\text { Net Profit }\end{array}$ & $\begin{array}{l}\text { Adjustment } \\
\text { Coefficient }\end{array}$ & $\begin{array}{l}\text { Lundberg's } \\
\text { Inequality }\end{array}$ \\
\hline \multirow{2}{*}{0.3} & $\begin{array}{l}a_{1}=01.00 \\
a_{2}=01.00\end{array}$ & \multirow{2}{*}{$\begin{array}{l}E\left[W_{1}\right]=1.3317 \\
E\left[W_{2}\right]=1.5803 \\
E[W]=2.9120\end{array}$} & $R_{1}=.01552$ & $\psi_{1}(30) \leq .6278$ \\
\hline & $\begin{array}{l}M_{1}=16.90 \\
M_{2}=01.34\end{array}$ & & $R_{2}=.1959$ & $\psi_{2}(15) \leq .0529$ \\
\hline \multirow[t]{2}{*}{0.4} & $\begin{array}{l}a_{1}=01.00 \\
a_{2}=01.00\end{array}$ & \multirow{2}{*}{$\begin{array}{l}E\left[W_{1}\right]=1.4583 \\
E\left[W_{2}\right]=1.5803 \\
E[W]=3.0387\end{array}$} & \multirow{2}{*}{$\begin{array}{l}R_{1}=.01508 \\
R_{2}=.1959\end{array}$} & $\psi_{1}(30) \leq .6361$ \\
\hline & $\begin{array}{l}M_{1}=22.31 \\
M_{2}=01.34\end{array}$ & & & $\psi_{2}(15) \leq .0529$ \\
\hline \multirow{2}{*}{0.5} & $\begin{array}{l}a_{1}=01.00 \\
a_{2}=01.00\end{array}$ & \multirow{2}{*}{$\begin{array}{l}E\left[W_{1}\right]=1.5101 \\
E\left[W_{2}\right]=1.5803 \\
E[W]=3.0904\end{array}$} & \multirow{2}{*}{$\begin{array}{l}R_{1}=.01495 \\
R_{2}=.1959\end{array}$} & $\psi_{1}(30) \leq .6386$ \\
\hline & $\begin{array}{l}M_{1}=27.12 \\
M_{2}=01.34\end{array}$ & & & $\psi_{2}(15) \leq .0529$ \\
\hline \multirow[t]{2}{*}{0.6} & $\begin{array}{l}a_{1}=01.00 \\
a_{2}=01.00\end{array}$ & \multirow{2}{*}{$\begin{array}{l}E\left[W_{1}\right]=1.5322 \\
E\left[W_{2}\right]=1.5803 \\
E[W]=3.1125\end{array}$} & \multirow{2}{*}{$\begin{array}{l}R_{1}=.01490 \\
R_{2}=.1959\end{array}$} & \multirow{2}{*}{$\begin{array}{l}\psi_{1}(30) \leq .6395 \\
\psi_{2}(15) \leq .0529\end{array}$} \\
\hline & $\begin{array}{l}M_{1}=31.54 \\
M_{2}=01.34\end{array}$ & & & \\
\hline
\end{tabular}


subsidize the payment of the reinsurance of those potentially more risky. In this example such interaction implied a decrease in the joint expected net profit, but there are substantial benefits in the company's security, as a whole. Nothing of this can be achieved, if one insists on treating each risk separately.

\section{REFERENCES}

Andreadakis, M. and Waters, H. R. (1980) The Effect of Reinsurance on the Degree of Risk Associated with an Insurance Portfolio. ASTIN Bulletin 11, 119-135.

Beard, R.E., Pentikainen, T. and Pesonen, E. (1984) Risk Theory, Chapman and Hall, London.

CENTENO, L. (1986) Measuring the effects of reinsurance by the adjustment coefficient. Insurance: Mathematics and Economics 5, 169-182.

Courant, R. and John, F. (1974) Introduction to Calculus \& Analysis, II. Wiley, London.

Gerber, H.U. (1979) An Introduction to Mathematical Risk Theory. S.S. Huebner Foundation, Philadelphia.

WATERS, H. R. (1979) Excess of Loss Reinsurance Limits. Scandinavian Actuarial Journal, 37-43.

WaTERS, H.R. (1983) Some Mathematical Aspects of Reinsurance. Insurance: Mathematics and Economics 2, 17-26.

\section{Maria de Lourdes Centeno}

Instituto Superior de Economia e Gestão, Rua Miguel Lupi, 20, 1200 Lisboa, Portugal. 